\title{
Factors Required during Preculture of Rat Oocytes Soon after Sperm Penetration for Promoting Their Further Development in a Chemically Defined Medium
}

\author{
Xin-Zhi YANG ${ }^{1)}$, Myung-Sook HAN ${ }^{1)}$, Koji NIWA ${ }^{1,2)}$ and \\ Philip M. IANNACCONE ${ }^{3)}$ \\ ${ }^{1)}$ Graduate School of Natural Science and Technology, Okayama University, Okayama 700- \\ 8530, ${ }^{2)}$ Faculty of Agriculture, Okayama University, Okayama 700-8530, Japan, \\ ${ }^{3)}$ Department of Pediatrics, Children's Memorial Institute for Education and Research, \\ Northwestern University Feinberg School of Medicine, 2300 Children's Plaza (204), Chicago, \\ Illinois 60614, USA \\ \#Present: Department of Animal Science, Jinju National University, Jinju 660-758, Korea
}

\begin{abstract}
Blastocyst formation in a chemically defined medium (mR1ECM) of rat oocytes soon after sperm penetration is less frequent than in those undergoing male pronuclear formation. This inhibition is released by preculturing the oocytes for a few hours in modified Krebs-Ringer bicarbonate solution (mKRB). The present study examined the effects of phosphate (Pi), bovine serum albumin (BSA) and osmolarity during preculture of sperm penetrated rat oocytes on their development to blastocysts in MR1ECM in vitro. These are the major factors that differ between $\mathrm{mR} 1 \mathrm{ECM}$ and $\mathrm{mKRB}$. When oocytes collected at $0730-0800 \mathrm{~h}$ on the day following mating and freed from cumulus cells were precultured for $5 \mathrm{~h}$ in $\mathrm{mKRB}$ or Pi-free $\mathrm{mKRB}$ and then cultured for $127 \mathrm{~h}$ in mR1ECM, about 73-74\% of oocytes developed to blastocysts. In both media, replacement of BSA with polyvinylalcohol (PVA) or osmolarity of $246 \mathrm{mOsM}$ reduced blastocyst formation compared with media containing BSA or with osmolarity of $304 \mathrm{mOsM}$; blastocyst formation was greatly inhibited when oocytes were precultured in media with PVA and osmolarity of $246 \mathrm{mOsM}$. On the other hand, when precultured in mR1ECM or mR1ECM with osmolarity of 304 mOsM or BSA instead of PVA, fewer oocytes developed to blastocysts than those precultured in Pi-free mKRB and mR1ECM with osmolarity of $304 \mathrm{mOsM}$ and BSA. These results indicate that both BSA and osmolarity, but not Pi, are essential factors during preculture of rat oocytes soon after sperm penetration for promoting their further development to blastocysts in a chemically defined medium.

Key words: Chemically defined medium, In-vitro development, One-cell embryo, Pronuclear formation, Rat
\end{abstract}

(J. Reprod. Dev. 50: 533-540, 2004)

D evelopment of chemically defined culture systems is important to the investigation of the factors involved in the in vitro development of embryos and to facilitate investigation of normal development of in vitro fertilized or manipulated

Accepted for publication: May 17, 2004

Correspondence: K. Niwa (e-mail: kniwa@cc.okayama-u.ac.jp) embryos. Robust in vitro preimplantation culture methods are important to embryo manipulation techniques and to transportation of zygotes. It was first reported that hamster embryos consistently developed beyond the 2-cell and 4-cell stages to the blastocyst stage in a chemically defined medium that did not contain glucose and phosphate (Pi) [1- 
3]. This new medium, designated as hamster embryo culture medium 1 (HECM-1) [1], was applied to the culture of rat $[4]$ and bovine $[5,6] 1$ cell embryos, although development to the blastocyst stage in this medium was limited. On the other hand, using HECM-1 without amino acids, it was found that the presence of $\mathrm{Pi}$ inhibited the development of rat 1-cell embryos beyond the 4-cell stage, but that the osmolarity of the medium and adequate concentrations of glucose are important factors for development to the blastocyst stage [7]. Thus, in HECM-1 modified by reducing $\mathrm{NaCl}$ concentration from $98.0 \mathrm{mM}$ to $78.8 \mathrm{mM}$, adding $7.5 \mathrm{mM}$ glucose, and omitting amino acids, about $60 \%$ of 1 -cell embryos collected from outbred Wistar rats developed to the blastocyst stage [7]. Therefore, the modified HECM-1 was designated as rat 1-cell embryo culture medium (R1ECM) [8]. It has been found that high proportions of rat 1-cell embryos produced in vivo [8,9] and in vitro [10] can develop to the blastocyst stage in R1ECM supplemented with 20 amino acids, and this chemically defined medium was designated as modified R1ECM (mR1ECM) [11, 12].

However, it has been reported that in hamsters development in a chemically defined medium, HECM-3, of 1-cell embryos collected from oviducts soon after sperm penetration, or obtained after in vitro fertilization, is difficult [13]. This inhibition of development has also been observed in rat 1-cell embryos before pronuclear formation collected from oviducts or produced by in vitro fertilization [12]. In rat embryos at early stages of sperm penetration, however, development was enhanced by preculturing them in modified Krebs-Ringer bicarbonate solution (mKRB) or by delaying the time of transfer from $m K R B$ to $m R 1 E C M$ in fertilization in vitro [12]. Furthermore, although 1cell embryos at the late stage of penetration collected from inbred strains of rats developed poorly or not at all in mR1ECM [9], they could successfully develop to the blastocyst stage in mR1ECM when precultured in KSOM [14], a medium used for culture of mouse implantation

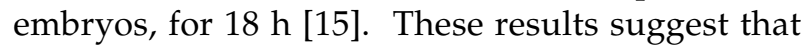
factors present (or absent) in mKRB or KSOM but absent (or present) in $\mathrm{mR} 1 \mathrm{ECM}$ are required during pronuclear formation, or even after pronuclear formation in inbred strains of rats, to achieve successful development to the blastocyst stage of rat 1-cell embryos in mR1ECM. The present study was undertaken to clarify key factors for the use of mKRB and mR1ECM as basic preculture media in a two phase in vitro culture system that supports development from rat 1-cell embryos, which were isolated soon after sperm penetration in vivo, to the blastocyst stage.

\section{Materials and Methods}

\section{Media}

Basic media used for preculture of 1-cell embryos were $m K R B$ and $m R 1 E C M$. The $m K R B$ was composed of $94.6 \mathrm{mM} \mathrm{NaCl}, 4.78 \mathrm{mM} \mathrm{KCl}, 1.71 \mathrm{mM}$ $\mathrm{CaCl}_{2}, 1.19 \mathrm{mM} \mathrm{KH} \mathrm{PO}_{4}, 1.19 \mathrm{mM} \mathrm{MgSO}_{4}, 25.07$ $\mathrm{mM} \mathrm{NaHCO} 3,21.58 \mathrm{mM}$ sodium lactate, $0.5 \mathrm{mM}$ sodium pyruvate, $5.56 \mathrm{mM}$ glucose, $4.0 \mathrm{mg} / \mathrm{ml}$ bovine serum albumin (BSA; A-7638; Sigma Chemical Co., St. Louis, MO, USA), $50 \mu \mathrm{g} / \mathrm{ml}$ streptomycin sulfate (S-9137; Sigma) and $75 \mu \mathrm{g} / \mathrm{ml}$ potassium penicillin-G (Sigma). This medium was essentially the same as that used by Toyoda and Chang [16] for in vitro fertilization of rat oocytes, except that phenol red was omitted. The mR1ECM was composed of $76.7 \mathrm{mM} \mathrm{NaCl}, 3.2 \mathrm{mM} \mathrm{KCl}, 2.0$ $\mathrm{mM} \mathrm{CaCl} 2,0.5 \mathrm{mM} \mathrm{MgCl}_{2}, 25.0 \mathrm{mM} \mathrm{NaHCO}, 10.0$ $\mathrm{mM}$ sodium lactate, $0.5 \mathrm{mM}$ sodium pyruvate, 7.5 $\mathrm{mM}$ glucose, $1.0 \mathrm{mg} / \mathrm{ml}$ polyvinylalcohol (PVA), 2\% (v/v) Minimal Essential Medium (MEM) essential amino acid solution (No. 11130-051; Gibco Laboratories, Grand Island, NY, USA), $0.1 \mathrm{mM}$ glutamine (Sigma) and 1\% (v/v) MEM nonessential amino acid solution (No. 11140-050; Gibco) [11, 12]. The mKRB with PVA $(1.0 \mathrm{mg} / \mathrm{ml})$ instead of BSA and $\mathrm{mR} 1 \mathrm{ECM}$ with BSA $(4.0 \mathrm{mg} / \mathrm{ml})$ instead of PVA were named mKRB-PVA and mR1ECM-BSA, respectively. The osmolarities of $\mathrm{mKRB}$ or $\mathrm{mKRB}$ PVA and mR1ECM or mR1ECM-BSA were about 304 and $246 \mathrm{mOsM}$, respectively, when determined by freezing point depression using the Fiske OneTen Osmometer (Fiske Association, Norwood, MA, USA). Culture medium for early development of precultured oocytes was mR1ECM. All preculture and culture media (each $400 \mu 1$ ) had been previously covered with paraffin oil (Nacalai Tesque Inc., Kyoto, Japan) in a polystyrene culture dish (35 mm $\times 10$ mm, No. 1008; Becton, Dickinson, Rutherford, NJ, USA) and equilibrated with the gas phase and temperature $\left(5 \% \mathrm{CO}_{2}\right.$ in air at $\left.37 \mathrm{C}\right)$ in a $\mathrm{CO}_{2}$ incubator for at least $4 \mathrm{~h}$. 


\section{Collection and treatment of oocytes}

Outbred Wistar rats were bred in-house and kept in a room under a 14L:10D photoperiod (lights-on, 0600-2000; lights-off, 2000-0600 h). Mature female rats (2-4 mo old) at proestrus, as assessed from vaginal smears, were placed overnight with mature males. At $0730-0800 \mathrm{~h}$ on the following day, the females were examined for mating by the presence of a vaginal plug or spermatozoa in the vagina. The oviducts were isolated and placed in a dish containing paraffin oil and each of the preculture media supplemented with $0.1 \%$ (w/v) hyaluronidase (No. H-3506; Sigma). The cumulusoocyte complexes were dissected out of the oviducts, placed in each medium, and freed from cumulus cell by repeated passage through a fine pipette.

\section{Culture of sperm penetrated oocytes and evaluation of embryo development}

The denuded oocytes were washed three times with each of the preculture media without hyaluronidase and observed for evidence of sperm penetration by means of a phase-contrast microscope. Only penetrated oocytes were washed again two times with each preculture medium, transferred (10-15 oocytes) into $400 \mu \mathrm{l}$ of the same medium, and precultured under $5 \% \mathrm{CO}_{2}$ in air at 37 $\mathrm{C}$ for $5 \mathrm{~h}$. Then the oocytes were transferred into $400 \mu \mathrm{l}$ of mR1ECM and cultured under the same temperature and atmospheric conditions. Oocytes were observed 27 and $127 \mathrm{~h}$ after the start of culture for their development to the 2-cell and blastocyst stages, respectively, using a phase-contrast microscope. Embryos showing blastocoele cavity formation were classified as blastocysts.

\section{Experiment 1}

To determine the effects of the presence of $\mathrm{Pi}$ in the preculture medium on development in mR1ECM, sperm penetrated oocytes were precultured for $5 \mathrm{~h}$ in $\mathrm{mKRB}$ with or without 1.19 $\mathrm{mM} \mathrm{KH}_{2} \mathrm{PO}_{4}$. The osmolarity of Pi-free mKRB was adjusted by increasing $\mathrm{NaCl}$ concentration from 94.6 to $95.8 \mathrm{mM}$.

\section{Experiment 2}

To determine the effects of BSA and/or osmolarity in preculture medium on the development in $\mathrm{mR} 1 \mathrm{ECM}$, penetrated oocytes were precultured for $5 \mathrm{~h}$ in $\mathrm{mKRB}$ or mKRB-PVA with or without Pi and with osmolarity of 304 or 246 mOsM. The osmolarity was adjusted to $246 \mathrm{mOsM}$ by reducing $\mathrm{NaCl}$ concentration from 94.6 to 66.1 $\mathrm{mM}$ and $67.3 \mathrm{mM}$ in $\mathrm{mKRB}$ and Pi-free mKRB, respectively.

\section{Experiment 3}

To determine the effects of BSA and/or osmolarity in mR1ECM instead of $\mathrm{mKRB}$ as preculture medium on the development in mR1ECM, penetrated oocytes were precultured for $5 \mathrm{~h}$ in mR1ECM or mR1ECM-BSA with osmolarity of 246 or $304 \mathrm{mOsM}$. The osmolarity was adjusted to $304 \mathrm{mOsM}$ by increasing $\mathrm{NaCl}$ concentration from 76.7 to $105.7 \mathrm{mM}$ in both media. As a control medium, Pi-free mKRB in which osmolarity was adjusted to $304 \mathrm{mOsM}$ by increasing $\mathrm{NaCl}$ concentration from 94.6 to $95.8 \mathrm{mM}$ was used.

\section{Experiment 4}

To determine whether osmolarity itself in preculture medium affects the development in mR1ECM, penetrated oocytes were precultured for $5 \mathrm{~h}$ in mR1ECM-BSA in which the osmolarity was adjusted to $304 \mathrm{mOsM}$ by increasing $\mathrm{NaCl}$ concentration from 76.7 to $105.7 \mathrm{mM}$ or by adding $58 \mathrm{mM}$ sorbitol.

\section{Statistical analysis}

Data obtained from four replicates were expressed as means \pm SEM. Statistical analyses for treatment comparisons were carried out by ANOVA and Duncan's multiple range test using the STATVIEW program (Abacus Concepts, Inc., Berkeley, CA, USA). All percentage data were subjected to an arc sine transformation before statistical analysis. When ANOVA revealed a significant treatment effect, the treatments were compared by Duncan's multiple range test.

\section{Results}

Effects of the presence of Pi in $m K R B$ (Experiment 1)

As shown in Table 1, most sperm penetrated oocytes precultured for $5 \mathrm{~h}$ in $\mathrm{mKRB}$ with and without Pi cleaved to the 2-cell stage $27 \mathrm{~h}$ after the start of culture in mR1ECM. The differences between the two preculture media were not statistically significant. Furthermore, at $127 \mathrm{~h}$ after the start of culture, there was not a significant 
Table 1. Effects of phosphate (Pi) in mKRB for preculture of rat oocytes just after sperm penetration on their further development in mR1ECM ${ }^{a}$

\begin{tabular}{cccc}
\hline & No. of oocytes & $\%($ mean \pm SEM) of embryos developed \\
\cline { 3 - 4 } $\mathrm{Pi}$ & cultured & 2-cell $(27)^{\mathrm{b}}$ & Blastocyst $(127)^{\mathrm{b}}$ \\
\hline+ & 51 & $96.2 \pm 3.8$ & $72.6 \pm 1.2$ \\
- & 51 & $90.1 \pm 1.9$ & $74.2 \pm 3.2$ \\
\hline
\end{tabular}

${ }^{a}$ Experiments were repeated 4 times.

${ }^{b}$ Numbers in parentheses indicate the time of examination (hours after the start of the second phase of culture in mR1ECM).

difference in the proportion of oocytes that developed to the blastocyst stage.

Effects of $B S A$ and/or osmolarity in $m K R B$ with or without Pi (Experiment 2)

As shown in Table 2, similar high proportions of oocytes cleaved to the 2 -cell stage $27 \mathrm{~h}$ after the start of the second phase of culture in mR1ECM, irrespective of the presence of $\mathrm{Pi}$ and/or BSA and of different osmolarities in $\mathrm{mKRB}$ for preculture of oocytes. However, the proportion of oocytes that developed to the blastocyst stage $127 \mathrm{~h}$ after the start of culture in mR1ECM was significantly $(\mathrm{P}<0.05)$ lower when sperm penetrated oocytes were precultured in $m K R B$ with reduced osmolarity (246 mOsM) or mKRB-PVA than in mKRB with osmolarity of $304 \mathrm{mOsM}$, irrespective of the presence of $\mathrm{Pi}$ in the media. The lowest proportion of blastocyst formation was obtained in oocytes precultured in mKRB-PVA with osmolarity of $246 \mathrm{mOsM}$ both in the presence and absence of $\mathrm{Pi}$ $(\mathrm{P}<0.05)$.

\section{Effects of BSA and/or osmolarity in mR1ECM (Experiment 3)}

As shown in Table 3, similar high proportions of oocytes cleaved to the 2 -cell stage $27 \mathrm{~h}$ after the start of the second phase of culture in $\mathrm{mR} 1 \mathrm{ECM}$, irrespective of the replacement of PVA with BSA or of different osmolarities in $\mathrm{mR} 1 \mathrm{ECM}$ for preculture of oocytes. When sperm penetrated oocytes were precultured in MR1ECM-BSA with osmolarity of $304 \mathrm{mOsM}$, a similar proportion of oocytes developed to the blastocyst stage to those precultured in control medium (Pi-free mKRB). However, blastocyst formation was significantly $(\mathrm{P}<0.05)$ inhibited when sperm penetrated oocytes were precultured in mR1ECM with osmolarity of $304 \mathrm{mOsM}$ or mR1ECM-BSA than in mR1ECMBSA with osmolarity of $304 \mathrm{mOsM}$. The lowest proportion of blastocyst formation was obtained in oocytes precultured in mR1ECM with osmolarity of 246 mOsM $(\mathrm{P}<0.05)$.

Effects of adjustment of osmolarity by adding $\mathrm{NaCl}$ or sorbitol in mR1ECM-BSA (Experiment 4)

As shown in Table 4, most sperm penetrated oocytes cleaved to the 2 -cell stage $27 \mathrm{~h}$ after the start of culture in mR1ECM following preculture for $5 \mathrm{~h}$ in mR1ECM-BSA or that with osmolarity of 304 mOsM adjusted by adding $\mathrm{NaCl}$ or sorbitol.

Table 2. Effects of osmolarity in mKRB or mKRB-PVA with or without phosphate (Pi) for preculture of rat oocytes just after sperm penetration on their further development in $\mathrm{mR}_{\mathrm{ECCM}}{ }^{\mathrm{a}}$

\begin{tabular}{|c|c|c|c|c|c|}
\hline \multirow[b]{2}{*}{$\mathrm{Pi}$} & \multirow[b]{2}{*}{ Medium } & \multirow{2}{*}{$\begin{array}{l}\text { Osmolarity } \\
(\mathrm{mOsM})\end{array}$} & \multirow{2}{*}{$\begin{array}{c}\text { No. of oocytes } \\
\text { cultured }\end{array}$} & \multicolumn{2}{|c|}{$\%$ (mean \pm SEM) of embryos developed } \\
\hline & & & & 2-cell $(27)^{\mathrm{b}}$ & Blastocyst $(127)^{\mathrm{b}}$ \\
\hline \multirow[t]{4}{*}{+} & mKRB & 304 & 57 & 100 & $74.4 \pm 2.5^{c}$ \\
\hline & & 246 & 57 & $84.2 \pm 4.0$ & $49.3 \pm 3.8^{\mathrm{d}}$ \\
\hline & mKRB-PVA & 304 & 56 & $91.9 \pm 4.3$ & $54.8 \pm 1.9^{\mathrm{d}}$ \\
\hline & & 246 & 57 & $91.0 \pm 3.4$ & $34.3 \pm 1.7^{\mathrm{e}}$ \\
\hline \multirow[t]{4}{*}{-} & $\mathrm{mKRB}$ & 304 & 41 & $94.7 \pm 3.1$ & $71.1 \pm 2.4^{\mathrm{c}}$ \\
\hline & & 246 & 41 & $90.7 \pm 6.4$ & $51.4 \pm 2.3^{\mathrm{d}}$ \\
\hline & mKRB-PVA & 304 & 41 & $90.7 \pm 3.7$ & $56.2 \pm 1.3^{\mathrm{d}}$ \\
\hline & & 246 & 40 & $89.9 \pm 0.4$ & $37.2 \pm 3.5^{\mathrm{e}}$ \\
\hline
\end{tabular}

a Experiments were repeated 4 times.

${ }^{\mathrm{b}}$ Numbers in parentheses indicate the time of examination (hours after the start of the second culture in $\mathrm{mR} 1 \mathrm{ECM}$ ).

c-e Values with different superscripts within each column in each group with or without phosphate differ significantly $(\mathrm{P}<0.05)$. 
Table 3. Effects of osmolarity in mR1ECM or mR1ECM-BSA for preculture of rat oocytes just after sperm penetration on their further development in $\mathrm{mR} \mathrm{ECM}^{\mathrm{a}}$

\begin{tabular}{|c|c|c|c|c|}
\hline \multirow[b]{2}{*}{ Medium } & \multirow{2}{*}{$\begin{array}{l}\text { Osmolarity } \\
(\mathrm{mOsM})\end{array}$} & \multirow{2}{*}{$\begin{array}{c}\text { No. of oocytes } \\
\text { cultured }\end{array}$} & \multicolumn{2}{|c|}{$\%($ mean \pm SEM) of embryos developed } \\
\hline & & & 2 -cell $(27)^{\mathrm{b}}$ & Blastocyst $(127)^{\mathrm{b}}$ \\
\hline Pi-free $\mathrm{mKRB}$ & 304 & 45 & $95.8 \pm 2.5$ & $73.2 \pm 1.4^{\mathrm{c}}$ \\
\hline \multirow[t]{2}{*}{ mR1ECM } & 304 & 43 & $95.3 \pm 2.8$ & $56.0 \pm 2.9^{e}$ \\
\hline & 246 & 42 & $90.5 \pm 0.7$ & $35.0 \pm 2.3^{\mathrm{d}}$ \\
\hline \multirow[t]{2}{*}{ mR1ECM-BSA } & 304 & 45 & $98.0 \pm 2.0$ & $73.8 \pm 2.4^{c}$ \\
\hline & 246 & 44 & $92.5 \pm 5.2$ & $52.5 \pm 2.5^{\mathrm{e}}$ \\
\hline
\end{tabular}

a Experiments were repeated 4 times.

${ }^{\mathrm{b}}$ Numbers in parentheses indicate the time of examination (hours after the start of the second culture in $\mathrm{mR} 1 \mathrm{ECM})$.

${ }^{c-e}$ Values with different superscripts within column differ significantly $(\mathrm{P}<0.05)$.

Table 4. Effects of preculture in mR1ECM-BSA with high osmolarity (310 mOsM) adjusted by adding $\mathrm{NaCl}$ or sorbitol of rat oocytes just after sperm penetration on their further development in $\mathrm{mR} 1 \mathrm{ECM}^{\mathrm{a}}$

\begin{tabular}{|c|c|c|c|c|}
\hline \multirow{2}{*}{$\begin{array}{c}\text { Osmolarity } \\
\text { (mOsM) }\end{array}$} & \multirow[b]{2}{*}{ Adjusted by ${ }^{b}$} & \multirow{2}{*}{$\begin{array}{c}\text { No. of oocytes } \\
\text { cultured }\end{array}$} & \multicolumn{2}{|c|}{$\%($ mean \pm SEM) of embryos developed } \\
\hline & & & 2 -cell $(27)^{b}$ & Blastocyst (127) ${ }^{\mathrm{b}}$ \\
\hline 246 & - & 51 & $96.2 \pm 2.1$ & $39.9 \pm 5.0^{c}$ \\
\hline 304 & $\mathrm{NaCl}$ & 51 & $98.3 \pm 1.7$ & $72.0 \pm 6.2^{\mathrm{d}}$ \\
\hline 304 & Sorbitol & 51 & $98.3 \pm 1.7$ & $74.2 \pm 2.3^{\mathrm{d}}$ \\
\hline
\end{tabular}

However, the incidence of blastocyst formation was significantly $(\mathrm{P}<0.05)$ higher in oocytes precultured in mR1ECM-BSA with osmolarity of 304 than 246 mOsM. There was no significant diffrence in blastocyst formation between oocytes precultuted in the medium with osmolarity of $304 \mathrm{mOsM}$ adjusted by increasing $\mathrm{NaCl}$ concentration and by adding sorbitol.

\section{Discussion}

As previously observed [12], about $40 \%$ of oocytes collected at $0730-0800 \mathrm{~h}$ on the day following mating were penetrated in the present study (data not shown). The penetrated oocytes had spermatozoa in the perivitelline space with intact sperm heads or had enlarged sperm heads within the oocyte cytoplasm. Since it was difficult to observe enlarged sperm heads within the cytoplasm without staining oocytes, only those oocytes in which a penetrating sperm tail was observed were considered penetrated with an enlarged intracytoplasmic sperm head. In the present study, these sperm penetrated oocytes were precultured for $5 \mathrm{~h}$ in $\mathrm{mKRB}$ or $\mathrm{mR} 1 \mathrm{ECM}$ that were variously modified, by removing $\mathrm{Pi}$, replacing BSA (PVA) with PVA (BSA), or changing osmolarity, because these are major factors that differ between mKRB and mR1ECM. When precultured oocytes were further cultured in mR1ECM for $27 \mathrm{~h}$, very high proportions (84-100\%) of oocytes cleaved to the 2-cell stage. This indicates that rat oocytes at the early stage of sperm penetration can undergo fertilization equally well in the various preculture media used in this study. However, the proportion of oocytes that developed to the blastocyst stage was significantly different for certain preculture media, indicating that the conditions during preculture of oocytes at the early stage of sperm penetration affect the in vitro developmental potential of the fertilized oocytes.

In the present study, when mKRB with or without Pi or mR1ECM-BSA with osmolarity of 304 
mOsM was used as preculture media, $71-74 \%$ of oocytes developed to the blastocyst stage. However, the incidence of blastocyst formation was reduced to $49-56 \%$ by preculture of oocytes in mKRB or Pi-free mKRB with osmolarity of 246 mOsM, mKRB-PVA or Pi-free mKRB-PVA, mR1ECM with osmolarity of $304 \mathrm{mOsM}$, and mR1ECM-BSA. These results indicate that both conditions, the presence of BSA, but not $\mathrm{Pi}$, and with osmolarity of $304 \mathrm{mOsM}$ are essential for rat oocytes just after sperm penetration to improve the ability to develop to the blastocyst stage in vitro in mR1ECM. On the other hand, it has been reported that oocytes even at the late 1-cell stage obtained from 4 inbred strains of rats developed poorly or not at all in mR1ECM [9]. However, when they were precultured in KSOM for $18 \mathrm{~h}$, they could successfully develop to the blastocyst stage in mR1ECM [15]. Although KSOM contains only 1 $\mathrm{mg} / \mathrm{ml} \mathrm{BSA}$ and its osmolarity is comparatively low (about $273 \mathrm{mOsM}$ ) [14], it seems that the requirement of BSA and higher osmolarity than that in mR1ECM during the early stage of penetration of oocytes for maintaining their further development to the blastocyst stage in $\mathrm{mR} 1 \mathrm{ECM}$ is adaptable to other strains of rats. However, since oocytes at the stage of syngamy were precultured in KSOM in the inbred strains of rats, the developmental stage of sperm penetrated oocytes requiring BSA and a fixed osmolarity may vary among the different strains of rats. On the other hand, the efficacy of blastocyst formation of sperm penetrated oocytes precultured in KSOM was largely different among different inbred strains of rats [15]. Furthermore, in oocytes at the pronuclear stage obtained from outbred Sprague-Dauley rats, the efficacy of blastocyst formation in $\mathrm{mR} 1 \mathrm{ECM}$ was also different according to the different preculture media [15]. These data suggest that the concentration of BSA and osmolarity required during preculture of oocytes also vary among different strains of rats.

When rat oocytes penetrated in vivo at the stage before male pronuclear formation were precultured for $4-6 \mathrm{~h}$ in media with BSA and osmolarity of 304 mOsM, $71-78 \%$ of them developed to the blastocyst stage in mR1ECM [12 and present study]. However, a comparatively high proportion (93\%) of oocytes developed to the blastocyst stage when those undergoing male pronuclear formation in vivo (1200-1300 $\mathrm{h}$ on the day following mating) were cultured directly in mR1ECM [12]. Thus it appears that some factors other than BSA and osmolarity are still lacking in $\mathrm{mKRB}$ or $\mathrm{mR} 1 \mathrm{ECM}$ BSA with osmolarity of 304 mOsM to compensate for the oviductal environment that maintains development of oocytes at the early stages preceding pronuclear formation. However, there was no difference in the ability to maintain the development of oocytes at the early stage between both media in the present experiments. It is also possible that BSA concentration and osmolarity used in the present study still may not be optimum. On the other hand, it has been reported in hamsters that the low development of oocytes soon after penetration in a chemically defined medium is overcome by supplementation of the medium with hypotaurine [13]. It might be interesting to determine in future experiments whether hypotaurine works similarly in rat oocytes at the early stage of sperm penetration.

The results of the present study suggest that the osmolarity, and not the $\mathrm{NaCl}$ concentration, is responsible for promoting the ability of oocytes penetrated at the early stage to develop to the blastocyst stage in mR1ECM. This is consistent with similar osmolarity requirements previously described for the transition of rat embryos in vitro from morula to blastocyst [17]. Even in mR1ECMBSA with a reduced concentration of $\mathrm{NaCl}(76.7$ $\mathrm{mM})$, the development of oocytes precultured in the medium to the blastocyst stage was not affected when the osmolarity of the medium was adjusted to $304 \mathrm{mOsm}$ by adding sorbitol. Since sorbitol is not metabolized by embryos and has no detrimental effect on embryonic development, it is used effectively to adjust the osmolarity of culture medium for pig embryos [18]. In the present study, we did not determine the optimal preculture osmolarity, but we have clearly shown here that preculture of sperm penetrated oocytes in media with osmolarity of $246 \mathrm{mOsM}$, but not $304 \mathrm{mOsM}$, reduces their subsequent development to blastocysts in mR1ECM. On the other hand, such low osmolarity can support the development of rat oocytes after pronuclear formation albeit with strain dependent variability $[8,9]$. Similarly, BSA is one of the essential factors for promoting the ability of rat oocytes at the early stage of sperm penetration to develop to the blastocyst stage in mR1ECM. However, BSA is not essential for the development of oocytes after pronuclear formation 
$[8,9]$, although it maintains the development of pronuclear stage oocytes as well as PVA in mR1ECM [19]. Further studies are needed to determine the optimum BSA concentration and osmolarity for supporting maximum possible rate of development, and to clarify the mechanism(s) by which osmolarity or BSA alters the in vitro developmental potential of sperm penetrated rat oocytes in a stage dependent manner.

We conclude that both BSA and osmolarity, but not $\mathrm{Pi}$, are essential factors during preculture of rat oocytes soon after sperm penetration for promoting subsequent development to blastocysts in vitro in a chemically defined medium. These data will enable a more complete understanding of the optimum conditions required for the development in vitro of preimplantation rat oocytes. Robust in vitro culture of preimplantation rat embryos is important to transportation of rat zygotes and to many longitudinal studies of nuclear transfer, cloning, zygotic gene expression studies and cyropreservation.

\section{Acknowledgment}

This work was supported in part by a grant from Takeda Chemical Industries, Ltd. (Osaka, Japan), by a Grant-in-Aid for for Creative Scientific Research from the Japan Society for the Promotion of Science (13GS0008) and by grant RR016939 from the US DHHD, NIH.

\section{References}

1. Schini SA, Bavister BD. Two-cell block to development of cultured hamster embryos is caused by phosphate and glucose. Biol Reprod 1988; 39: 1183-1192.

2. McKiernan SH, Bavister BD. Environmental variables influencing in vitro development of hamster 2-cell embryos to the blastocyst stage. Biol Reprod 1990; 43: 404-423.

3. Seshagiri PB, Bavister BD. Relative developmental abilities of hamster 2- and 8-cell embryos cultured in hamster embryo culture medium-1 and -2. J Exp Zool 1991; 257: 51-57.

4. Kishi J, Noda Y, Narimoto K, Umaoka Y, Mori T. Block to development in cultured rat 1-cell embryos is overome using medium HECM-1. Hum Reprod 1991; 6: 1445-1448.

5. Pinyopummintr T, Bavister BD. In vitro-matured/ in vitro-fertilized bovine oocytes can develop into morulae/blastocysts in chemically defined, proteinfree culture media. Biol Reprod 1991; 45: 736-742.

6. Kim J-H, Niwa K, Lim J-M, Okuda K. Effects of phosphate, energy substrates, and amino acids on development of in vitro-matured, in vitro-fertilized bovine oocytes in a chemically defined, protein-free culture medium. Biol Reprod 1993; 48: 1320-1325.

7. Miyoshi K, Funahashi H, Okuda K, Niwa K. Development of rat one-cell embryos in a chemically defined medium: effects of glucose, phosphate and osmolarity. J Reprod Fertil 1994; 100: 21-26.

8. Miyoshi K, Abeydeera LR, Okuda K, Niwa K. Effects of osmolarity and amino acids in a chemically defined medium on development of rat one-cell embryos. J Reprod Fertil 1995; 103: 27-32.

9. Iannaccone P, Taborn G, Garton R.
Preimplantation and postimplantation development of rat embryos cloned with cumulus cells and fibroblasts. Zygote 2001; 9: 135-143.

10. Oh SH, Miyoshi K, Funahashi H. Rat oocytes fertilized in modified rat 1-cell embryo culture medium containing a high sodium chloride concentration and bovine serum albumin maintain developmental ability to the blastocyst stage. Biol Reprod 1998; 59: 884-889.

11. Miyoshi K, Tanaka N, Niwa K. Penetration in vitro of naturally ovulated rat eggs and development of eggs in a chemically defined medium. J Mamm Ova Res 1995; 12: 35-39.

12. Miyoshi K, Kono T, Niwa K. Stage-dependent development of rat 1-cell embryos in a chemically defined medium after fertilization in vivo and in vitro. Biol Reprod 1997; 56: 180-185.

13. Barnett DK, Bavister BD. Hypotaurine requirement for in vitro development of golden hamster one-cell embryos into morulae and blastocysts, and production of term offspring from in vitro-fertilized ova. Biol Reprod 1992; 47: 297-304.

14. Lawitts JA, Biggers JD. Culture of preimplantation embryos. In: Wassarman PM, DePamphils ML (eds), Methods in Enzymology, Vol. 225 (Guide to Techniques in Mouse Development). San Diego, CA: Academic Press; 1993: 153-164.

15. Zhou Y, Galat V, Garton R, Taborn G, Niwa K, Iannaccone $\mathbf{P}$. Two-phase chemically defined culture system for preimplantation rat embryos. Genesis 2003; 36: 129-133.

16. Toyoda Y, Chang MC. Fertilization of rat eggs in vitro by epididymal spermatozoa and the development of eggs following transfer. J Reprod 
Fertil 1974; 36: 9-22.

17. Van Winkle LJ, Iannaccone PM, Campione AL, Garton RL. Transport of cationic and zwitterionic amino acids in preimplantation rat conceptuses. Dev Biol 1990; 142: 184-193.

18. Beckmann LS, Day BN. Effect of media $\mathrm{NaCl}$ concentration and osmolarity on culture of the early stage porcine embryo and viability of embryos cultured in a selected superior medium. Theriogenology 1993; 39: 611-622.

19. Han M-S, Niwa K. Effects of BSA and fetal bovine serum in culture medium on development of rat embryos. J Reprod Dev 2003; 49: 235-242. 